\title{
Erratum
}

\section{Markov Partition for Dispersed Billiards}

\author{
L. A. Bunimovich and Ya. G. Sinai \\ L. D. Landau Institute for Theoretical Physics, ul. Kosygina, dom 2, SU-117334 Moscow, V-334, \\ USSR
}

Commun. Math. Phys. 78, 247-280 (1980)

The aim of this note is to make some changes in the proof of Doeblin's condition for Markov partitions constructed in the entitled paper. We used the assertion about the existence of a finite collection $A=\left(C_{1}(\eta), \ldots, C_{m_{0}}(\eta)\right)$ of elements of the Markov partition $\eta$ such that for every $C(\eta) \notin \bigcup_{i=1}^{m_{0}} C_{i}(\eta)$ the set $T C(\eta)$ is another element of $\eta$. This statement in its exact form is wrong. Instead one should use the following proposition.

First introduce some definitions. Let a number $c>0$ be such that each regular segment of 1.u.t.f. $\gamma^{(u)}\left(\right.$ l.s.t.f. $\left.\gamma^{(s)}\right)$ with the length less than $c$ intersects not more than $r$ curves of the set $\bigcup_{i=1}^{K_{0}} T^{-i} S_{0}\left(\bigcup_{i=1}^{K_{0}} T^{i} S_{0}\right), \Lambda_{\min }^{K_{0}}>r+1$, and there exists an integer $K_{1}$ such that $\max _{C_{\xi_{p}^{(u), p}>K_{1}}}\left(\operatorname{length}\left(C_{\xi_{p}^{(u)}}\right)\right)<c, \max _{C_{\xi^{(u)}}}\left(\right.$ length $\left(C_{\left.\xi_{p}^{(u)}\right)}\right)>c$, where $\xi_{p}^{(u)}=\xi^{(u)} \vee \eta_{p}$. Let $A_{n_{1}, n_{2}}$ be the set of all elements $C_{i}=C_{i}(\eta)$ for which $r_{+}(i) \leqq n_{1}, r_{-}(i) \leqq n_{2}$. We shall introduce also the following sets

$$
\begin{gathered}
G_{n,+}^{K_{1}}=\left\{x: T_{1}^{s} x \in A_{K_{1}, \infty} \text { for some } s=0,1, \ldots, n\right\}, \\
B_{n,+}^{K, K_{1}}=A_{K, \infty} \cap G_{n,+}^{K_{1}},
\end{gathered}
$$

and

$$
F_{n,+}^{K, K_{1}}\left(\alpha_{1}\right)=\left\{C_{\tilde{\eta}_{K}^{(u)}}: v\left(B_{n,+}^{K, K_{1}} / C_{\widetilde{\eta}_{K}^{(u)}}\right)>1-\alpha_{1}^{n y^{y}}\right\},
$$

where $0<\alpha_{1}<1, \gamma>0$, and $\tilde{\eta}_{K}^{(u)}$ is the partition of the set $A_{K, \alpha}$ induced by $\eta^{(u)}$.

Proposition. There exist numbers $\alpha_{1}, C>0, \gamma, \gamma^{\prime}>0$ and $\delta, 0<\delta<1$, such that for all $n>0$,

$$
v\left(\bigcup_{K=1}^{n} \bar{F}_{n, \infty}^{K, K_{1}}\left(\alpha_{1}\right)\right)<C \delta^{n \gamma^{\prime}}
$$

where $\bar{F}$ denotes a complement of a set $F$. 
Proof. We introduce for any integer $n>0$ and some $\delta_{1}, 0<\delta_{1}<1$, the following sets

$$
\begin{gathered}
\mathscr{D}_{n}^{K}\left(\delta_{1}\right)=\left\{x: x \in C_{\xi_{K}^{(u)}}, \text { length }\left(C_{\xi_{K}^{(u)}}\right) \leqq \delta_{1}^{n}, K \leqq n\right\}, \\
\mathscr{D}^{K}(c)=\left\{x: x \in C_{\xi_{K}(u)}, \text { length }\left(C_{\xi_{K}^{(u)}}\right)>c\right\} .
\end{gathered}
$$

It follows from the general theory of dispersed billiards [1] that there exists a constant $\delta_{2}, \delta_{1}<\delta_{2}<1$, such that $v\left(\bigcup_{K=1}^{n} \mathscr{D}_{n}^{K}\right)<\delta_{2}^{n}$.

Let us consider now the following set

$$
\hat{\mathscr{D}}_{n}^{K}=\left\{x: x \notin \mathscr{D}_{n}^{K}, x \in C_{\xi_{K}(u)}, T_{1}^{i} x \notin \mathscr{D}(c), i=0,1, \ldots, n\right\} .
$$

We shall show that there exist positive numbers $\beta_{1}, \beta_{2}<1$ and $\gamma_{1}, \gamma_{2}>0$ such that

$$
v\left\{x: x \in C_{\xi_{K}^{(u)}} C \overline{\mathscr{D}}_{n}^{K}\left(\delta_{1}\right), v\left(\hat{\mathscr{D}}_{n}^{K} / C_{\xi_{K}^{(u)}}\right)>\beta_{1}^{n \gamma_{1}}\right\}<\beta_{2}^{n \gamma_{2}} .
$$

Really because of the choice of $r, K_{1}$, and $\mathscr{D}_{n}^{K}\left(\delta_{1}\right)$ the set $T_{1}^{n} C_{\xi(u)}$ consists of not more than $(r+1)^{n}$ connected components. Let $x \notin \mathscr{D}_{n}^{K}$. Then $\Lambda_{\min }^{n K_{0}}$ length $\left(C_{\xi_{\xi}(u)}\right)$ $>\Lambda_{\min }^{n K o} \delta_{1}^{n}$. The sum of lengths of all such connected components $C$ of the set $T_{1}^{n} C_{\xi_{(u)}^{K}}^{K}$ for which $T_{1}^{-s} C \notin \mathscr{D}^{K}(c)$ for $s=0,1, \ldots, n$ is not more than $c A_{\min }^{-n K_{0}}(r+1)^{n}$. Therefore in view of the definition of $\delta_{1}$ the conditional measure on $C_{\xi_{K}(u)}$ of the preimage (under $T_{1}^{-n}$ ) of the union of all these components is less than $C_{1}$ (length $\left.\left(C_{\left.\xi_{(u}\right)}\right)\right)^{-1} A_{\min }^{-n K_{0}}(r+1)^{n}<c A_{\min }^{-n K_{0}}(r+1)^{n} \delta_{1}^{-n}$. Now the inequality (1) holds if we take such $\delta_{1}$ that $(r+1) \Lambda_{\min }^{-K_{0}} \delta_{1}^{-1}<1$. Besides we have that $(r+1) \Lambda_{\min }^{-K_{0}} \delta_{1}^{-1}$ $<\beta_{1}<1$ and $\beta_{1}<\beta_{2}<1$.

So we have the proposition for elements of the partition $\xi_{K}^{(u)}$, i.e. for connected pieces of l.u.t.f. Making use of the construction of the Markov partition $\eta$ one can derive using the analogous considerations that this proposition holds for elements of the partition $\tilde{\eta}_{K}^{(u)}$ as well. Now the proof of Doeblin's condition goes in the same way as in the entitled paper.

\section{References}

1. Sinai, Ya.G.: Dynamical systems with elastic reflections. Russ. Math. Surv. XXV, No. 2, 141-192 (1970)

Communicated by A. Jaffe

Received July 22, 1986 